\title{
Histomorphological spectrum of masses of the nasal cavity, paranasal sinuses and nasopharynx
}

\author{
Parajuli $\mathrm{S}^{1}$, Tuladhar $\mathrm{A}^{1}$

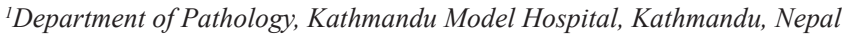

\section{Keywords: \\ Nasal Cavity; Nasopharynx; Paranasal Sinuses}

\begin{abstract}
Background: Swellings of nasal cavity, paranasal sinuses and nasopharynx have inflicted man from time immemorial. Nose is the most prominent part of the face with substantial aesthetic and functional significance. A variety of non-neoplastic and neoplastic conditions involve the nasal cavity, paranasal sinuses and nasophaynx, and these are very common lesions encountered in clinical practice.
\end{abstract}

Materials and Methods: This retrospective cross-sectional study was done in the Department of Pathology, Kathmandu Model Hospital for a period of 5 years from August 2007 to August 2012. Demographic data including age, sex and clinical presentation were obtained from the histopathology records.

Results: A total of 148 samples of tissue sent for histopathology were studied, out of which 119 were nonneoplastic and 29 were neoplastic lesions. A slight female preponderance was observed. Inflammatory nasal polyp was the most common non-neoplastic lesions encountered accounting for a total of 106 cases (89\%). Similarly, inverted papilloma was the most common benign lesion (6 cases) and nasopharyngeal carcinoma was the most common malignant lesion ( 5 cases).

Conclusion: Sino-nasal masses have various differential diagnoses. Malignancy should be distinguished from non-malignant lesions. A detailed history, clinical examination and most importantly, thorough histopathological evaluation are essential components of workup of patients for timely medical intervention

\section{INTRODUCTION}

Nasal masses are a common finding in an ENT (Ear, Nose and Throat) outpatient department. Nasal obstruction is the most common symptom. Other symptoms include nasal discharge, epistaxis and disturbances of smell. ${ }^{1}$

A variety of non-neoplastic including congenital and inflammatory lesions and neoplastic conditions involve the

\section{Correspondence:}

Dr. Sharmila Parajuli, $M D$

Department of Pathology, Kathmandu Model Hospital, Kathmandu, Nepal. E-mail:drsharmi@hotmail.com nasal cavity (NC), paranasal sinuses (PNS) and nasopharynx (NP) and these are very common lesions encountered in clinical practice. ${ }^{2}$

Gliomas are a common congenital sinonasal masses. Nasal polyps result from chronic inflammation of the nasal and sinus mucous membranes and are the most common tumors of the nasal cavity. Their exact pathogenesis is not known, however a strong association with allergy, infection, asthma and aspirin sensitivity has been implicated. Nasal polyps are a common cause of nasal obstruction in adults with a prevalence of about $4 \%$ in the general population.,

The presenting symptoms of all tumors are similar and using 
advanced imaging and computed tomography (CT) and/or magnetic resonance imaging (MRI), presumptive diagnosis are often made. However, a careful histopathological examination is necessary to decide the nature of any particular lesion including malignancy. Even though the malignant lesions have low incidence, they have a long clinical history with frequent local recurrence and they cause a relatively great amount of morbidity. Histological examination of these lesions is the gold standard for diagnosis because management and prognosis vary among different lesions. ${ }^{5,6}$

The aim of this study was to compare the incidence of non-neoplastic and neoplastic masses of the NC, PNS and NP and to list the different types of non-neoplastic and neoplastic lesions in our hospital.

\section{MATERIALS AND METHODS}

This retrospective cross-sectional study was done in the Department of Pathology, Kathmandu Model Hospital for a total duration of 5 years between August 2007 and August 2012. Demographic data including age, sex and clinical presentation were retrieved from the histopathology records.

All patients diagnosed with masses of the nasal cavity, paranasal sinuses and nasopharynx were included. The criteria for selection of cases were mainly based on history and clinical examination. Detailed history was taken considering the patient's complaints, mainly nasal obstruction, mass in the nose, epistaxis, rhinorrhoea, hyposmia and deformity of nose and face. Occupational history, personal habits and socioeconomic status of patients were documented. Clinical examinations were carried out as per standard protocols. Necessary radiological and laboratory investigations were done. Biopsies were taken from all cases for histopathological examination to confirm the diagnosis.

The biopsy samples were received in $10 \%$ formalin, routinely processed for histopathological examination and stained by Hematoxylin and Eosin (H \& E).

Table 1: Incidence of neoplastic and non-neoplastic lesions according to gender

\begin{tabular}{|c|c|c|c|c|}
\hline \multicolumn{2}{|c|}{ Type of mass } & Male & Female & Total \\
\hline \multicolumn{2}{|l|}{$\begin{array}{l}\text { Non-neoplastic } \\
\text { lesions }\end{array}$} & 50 & 69 & 119 \\
\hline \multirow[t]{2}{*}{$\begin{array}{l}\text { Neoplastic } \\
\text { lesions }\end{array}$} & Benign & 8 & 11 & 19 \\
\hline & Malignant & 6 & 4 & 10 \\
\hline \multicolumn{2}{|l|}{ Total } & 64 & 84 & 148 \\
\hline
\end{tabular}

Table 2: Distribution of lesions according to age

\begin{tabular}{ccccc}
\hline $\begin{array}{c}\text { Age } \\
\text { (Years) }\end{array}$ & $\begin{array}{c}\text { Non-neoplastic } \\
\text { lesions }\end{array}$ & Benign & Malignant & Total \\
\hline$<10$ & 18 & 0 & 3 & 21 \\
$11-20$ & 34 & 3 & 0 & 37 \\
$21-30$ & 33 & 2 & 0 & 35 \\
$21-40$ & 19 & 2 & 1 & 22 \\
$41-50$ & 11 & 5 & 2 & 18 \\
\hline $51-60$ & 2 & 5 & 2 & 9 \\
$>60$ & 2 & 2 & 2 & 6 \\
\hline Total & $119(80 \%)$ & $19(12.83 \%)$ & $10(6.75 \%)$ & $148(100 \%)$ \\
\hline
\end{tabular}

Table 3: Histopathological diagnosis of non-neoplastic lesions

\begin{tabular}{lcc}
\hline $\begin{array}{c}\text { Non neoplastic } \\
\text { lesions }\end{array}$ & Incidence (Cases) & Percentage (\%) \\
\hline Nasal polyp & 106 & $89 \%$ \\
Rhinosporidiosis & 4 & $3.36 \%$ \\
Mucocele & 3 & $2.52 \%$ \\
Glial heterotopia & 2 & $1.68 \%$ \\
Lupus vulgaris & 4 & $3.36 \%$ \\
\hline \multicolumn{1}{c}{ Total } & 119 & $100 \%$ \\
\hline
\end{tabular}

Table 4: Histopathological diagnosis of benign neoplastic lesions

\begin{tabular}{lcc}
\hline \multicolumn{1}{c}{ Benign lesions } & Incidence & Percentage \\
\hline Capillary haemangioma & 5 & $26.31 \%$ \\
\hline Inverted papilloma & 6 & $31.57 \%$ \\
\hline Nasopharyngeal angiofibroma & 3 & $15.78 \%$ \\
\hline Ossifying fibroma & 2 & $10.52 \%$ \\
\hline Cemento-ossifying fibroma & 1 & $5.26 \%$ \\
\hline Neurofibroma & 2 & $10.52 \%$ \\
\hline Total & 19 & $100 \%$ \\
\hline
\end{tabular}

\section{RESULTS}

A total of 148 representative biopsy samples were examined in the 5 year period, which formed $7.6 \%$ of the total specimens received for histopathology. A slight female preponderance was observed and the male to female ratio was 1:1.31. Nonneoplastic lesions composed the majority of cases followed by benign neoplastic lesions (Table 1). Non-neoplastic lesions were most common in the age group of 11-20 years. The benign and malignant lesions were most common in the age group of 41-60 years and $>40$ years respectively (Table 2). Amongst the non-neoplastic leisons, nasal polyp was the most common comprising 106 cases (89\%),followed by 4 cases $(3.36 \%)$ each of Rhinosporidiosis and Lupus vulgaris (Table 3 ). As shown in Table 4, inverted papilloma was the most common benign neoplasm comprising 6 cases $(31.57 \%)$, followed by 5 cases $(26.31 \%)$ of capillary hemangioma and 3 cases $(15.78 \%)$ of nasopharyngeal 
Table 5: Histopathological diagnosis of malignant neoplastic lesions

\begin{tabular}{lcc}
\hline \multicolumn{1}{c}{ Malignant lesions } & Incidence & Percentage \\
\hline $\begin{array}{l}\text { Nasopharyngeal carcinoma of } \\
\text { keratinizing type }\end{array}$ & 2 & $20 \%$ \\
$\begin{array}{l}\text { Nasopharyngeal undifferentiated } \\
\text { carcinoma }\end{array}$ & 3 & $30 \%$ \\
Lymphoma & 3 & $30 \%$ \\
Olfactory neuroblastoma & 1 & $10 \%$ \\
Alveolar rhabdomyosarcoma & 1 & $10 \%$ \\
\hline \multicolumn{1}{c}{ Total } & 10 & $100 \%$ \\
\hline
\end{tabular}

angiofibroma. Nasopharyngeal undifferentiated carcinoma and lymphoma was the commonest amongst the malignant neoplasms comprising 3 cases (30\%), followed by 2 cases (20\%) of nasopharyngeal carcinoma of keratinizing type (Table 5).

\section{DISCUSSION}

Sinonasal masses had predilection for females, demonstrating a male to female ratio of 1:1.31 similar to a study done from Nigeria. ${ }^{7}$ An opposite ratio showing male preponderance- 1.7:1 was demonstrated by Zafar et al. from India. ${ }^{8}$

The $2^{\text {nd }}$ and $4^{\text {th }}$ decades of life are the most vulnerable period for development of sinonasal masses. Bakari et al. ${ }^{7}$ had reported a peak incidence of 33 years, while for Zafar et al. ${ }^{8}$ the mean age of presentation was 22.5 years. Malignancies have been reported generally after the fourth decade of life.

Non-neoplastic lesions formed $80 \%$ of the total cases of NC, PNS and NP in our study. Such a high proportion of nonneoplastic lesions have been reported in previous studies. ${ }^{7,9}$ Zafar U et al, ${ }^{8}$ conducted a study over a period of seven years in the department of Pathology and Otolaryngology at Jawaharlal Nehru Medical College, Aligarh and revealed that non-neoplastic lesions outnumber the neoplastic lesions with a percentage of $89 \%$ which is similar to our study.

Nasal polyps are the most common tumors of the nasal cavity. The incidence of nasal polyp was slightly higher in this study $(89 \%)$ as compared to the observations by Tondon et al. (64\%) ${ }^{6}$ and Anjali et al. (62.5\%). ${ }^{2}$ Bakari A et al. ${ }^{7}$ revealed simple inflammatory polyp as the commonest histological pattern.

Rhinosporodiosis, an endemic disease in Asia and a few African nations was also diagnosed and treated. We found 4 cases of rhinosporiodosis (fig. 1) accounting for 3.36\% amongst the non-neoplastic lesions, while Pradhananga et al. ${ }^{9}$ had encountered only one case $(0.69 \%)$ during two year

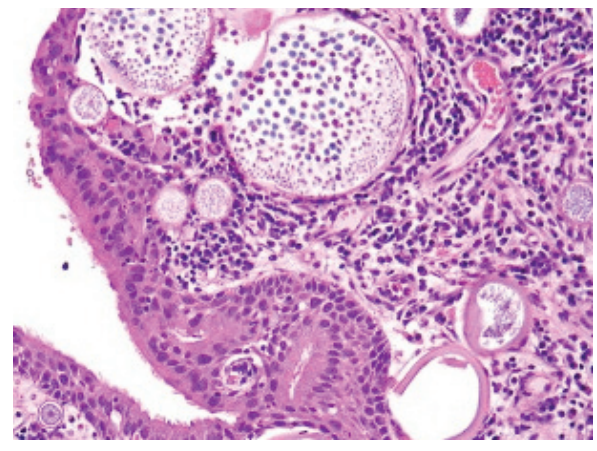

Figure 1: Rhinosporodiosis. Multiple thick walled sporangia are seen in the stroma containing numerous spores. (HE stain, X400).

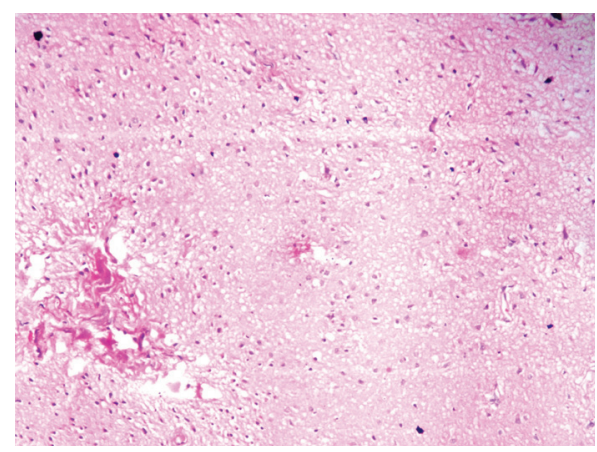

Figure 2: Glial heterotopia in nasal cavity showing mature glial tissue. (HE stain, X100).

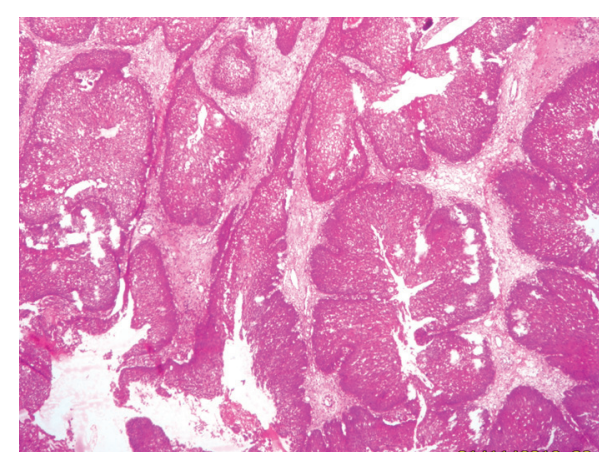

Figure 3: Inverted papilloma with typical inverted pattern of growth. (HE stain, X400).

study period. Anjali et al. ${ }^{2}$ analysed polypoidal lesions over a period of ten years and concluded that next to nasal polyp, rhinosporiodosis was the second common cause of nonneoplastic lesions. ${ }^{2}$

Nasal glial heterotopia (NGH) is a congenital malformation of displaced, mature glial tissue (choristomas) in which continuity with the intracranial meningeal component has become obliterated. Nasal glioma is a rare lesion and accounted for a total of $1.68 \%$ ( 2 cases; fig.2) amongst the non-neoplastic lesions in our study. Both the cases diagnosed in our study were below 1 year of age. According to Wischneiwski et al. ${ }^{10}$ glioma most commonly presents in newborn infants and children with features of respiratory 
abnormalities. Rahbor et $\mathrm{al}^{11}$ in their review article studied 10 cases of nasal glioma with the mean age of presentation as 9 months. The clinical and histological features in this case correlated with the study conducted by Chang et al. ${ }^{12}$ Clinically, the masses were firm, noncompressible, nonpulsatile gray or purple lesions that obstruct the nasal cavity intranasally and cause deformity extranasally. Histologically, these tumors were made up of astrocytic neuroglial cells interlaced with fibrous and vascular connective tissue that is covered with skin or nasal respiratory mucosa.

Three cases of mucocele were diagnosed in the present study accounting for a percentage of $2.52 \%$ amongst the nonneoplastic lesions. Mucoceles are chronic, cystic lesions in the paranasal sinuses. Similar incidence was reported in a retrospective review of the archives of the Singapore General Hospital otolaryngology department (1999-2006) which identified 10 cases of sphenoid sinus mucocele over a period of 7 years and found an incidence of $1-2 \% .{ }^{13}$

We diagnosed 5 cases $(26.31 \%)$ of capillary hemangioma amongst the benign lesions studied. Hemangioma is not regularly seen in the nasal cavity, though if it occurs, is predominantly capillary and is found attached to the nasal septum. All the cases were found to be arising from the cartilaginous part of the nasal septum. This finding corresponds to the observation of Pradhananga et al. ${ }^{10}$

We observed 4 cases (3.36\%) of lupus vulgaris (tuberculosis) but according to studies done by Waldman et al. ${ }^{14}$ and Nayar et al. ${ }^{15}$ sino-nasal tuberculosis are a rare entity. These differences might be due to the higher prevalence of tuberculosis in this part of the world. Acid fast bacilli stain and culture were positive.

Inverted papillomas are comparatively rare, but this morphological variant is the most commonly encountered lesion of all sinonasal neoplasms. ${ }^{16}$ Inverted papilloma (fig.3) formed $31.5 \%$ of all benign neoplastic masses in our study, which was similar to a study done by Humayun et al1 who reported $33.33 \%$ and marginally higher from the findings of Bakari et al. ${ }^{7}$ who reported as $14.5 \%$ amongst all the sinonasal masses. The rate of malignant transformation may be as high as $11 \% .{ }^{17}$ Inverted papilloma was associated with squamous cell carcinoma of the sinonasal cavity in 6 $(21.4 \%)$ of the 28 cases studied by Califano et al. ${ }^{18}$

Juvenile angiofibroma forms $0.5 \%$ of all head and neck tumors in Europe. ${ }^{19}$ We have reported 3 cases (10.34\%) of angiofibroma. All the cases were located in NP with profuse recurrent epistaxis from the polypoidal mass as the chief complaint. Pradhananga et al..$^{10}$ reported 9 cases $(6.29 \%)$ of angiofibroma over a period of 2 years in Nepal. It was the commonest benign tumor in a study by Khan $\mathrm{N}^{8}$ which accounted for $42.85 \%$ of all benign lesions.
Lesions with fibrous and osseous components include fibrous dysplasia, ossifying fibroma, and cemento-ossifying fibroma. We found two cases of ossifying fibroma and one case of cemento-ossifying fibroma accounting for a total of $10.52 \%$ and $5.26 \%$ respectively amongst the neoplastic benign lesion. Both the cases of ossifying fibroma were in the 1st decade of life with no sex predilection. The rarity of this lesion is documented by Choi et al. ${ }^{20}$ Lawton et al. ${ }^{21}$ studied four cases of ossifying fibroma, in which males predominated the picture and the mean age of presentation was 28 years. According to Jayachandran and Meenakshi, ${ }^{22}$ cemento-ossifying fibroma is a rare benign, non-odontogenic tumor-like lesion of the jaw with the age of occurrence between 20-40 years with female- to- male predilection of 2:1.

We present two cases (10.52\%) of neurofibroma of the nasal cavity treated by endoscopic surgery which were proven histologically. These tumors usually present as a solitary lesion and are difficult to diagnose before operation and histopathological examination.

Nasopharyngeal carcinoma is the most common cancer originating in the nasopharynx. We diagnosed 3 cases of undifferentiated carcinoma (30\%) and 2 cases of squamous cell carcinoma of keratinizing type $(20 \%)$. These tumors were rarely encountered before the $4^{\text {th }}$ decade of life. It formed $11.6 \%$ of all lesions and $40.6 \%$ of neoplastic lesions in a study done by A Lathi et al. ${ }^{23}$ Svane -Knudsen et al. ${ }^{24}$ have reported squamous cell carcinoma to be the most commonly encountered malignancy of the sinonasal tract in Denmark accounting for a total percentage of 35.65\% amongst all the sino-nasal malignancies. Pradhananga et al. $^{10}$ has reported $6.3 \%$ of their sinonasal masses to be malignant, while for Fasunla et al. ${ }^{25}$, malignant sinonasal tumors constituted $59.4 \%$ of the 138 sinonasal neoplasms seen.

We reported 3 cases of sinonasal lymphomas accounting for a total of $10.34 \%$ of the malignant neoplasms. The records of 23 patients diagnosed and treated at the Massachusetts General Hospital for extranodal non-Hodgkin's lymphoma of the paranasal sinus and nasal cavity were reviewed. ${ }^{26}$ Eight patients had nasal-type NK/T cell and 15 patients had diffuse large B-cell lymphoma.

Olfactory neuroblastoma is an uncommon neuroectodermal tumor that originates from the olfactory sensory epithelium in the upper nasal fossa at the level of the cribriform plate. We reported a single case of olfactory neuroblastoma accounting for $10 \%$ of the malignant tumors diagnosed. A study conducted in Japan revealed only 24 cases over a 18 year study period from 1964 to $1982 .{ }^{27}$ The average age of these patients is 42.3 years, ranging from 4 to 75 years old whereas the age group of the patient diagnosed in our study was below 10 years. This could probably be because this tumor is difficult to diagnose correctly, and the chance to 
start treatment early is missed at times.

Alveolar rhabdomyosarcoma of the head and neck is very rare in adults. We report a single case of alveolar rhabdomyosarcoma in the nasal cavity, occuring in a 24-year-old male patient presenting with left nasal obstruction and multiple neck nodules accounting for $10 \%$ of all the malignant tumors diagnosed. Rhabdomyosarcoma should be considered in the differential diagnosis of poorly defined soft tissue mass of the nasal cavity not only in children, but also in adults. Ahmed et al. ${ }^{28}$ reported 14 sinonasal rhabdomyosarcoma with an age range of 9 to 40 , out of which all of them were alveolar rhabdomyosarcoma except one which was embryonal type.

\section{REFERENCES}

1. Humayun AHM, Zahurul Huq AHM, Ahmed SMT et al. Clinicopathological study of sinonasal masses. Bangladesh J Otorhinolaryngol 2010;16:15-22.

2. Dasgupta A, Ghosh RN, Mukherjee C. Nasal polyps-histopathological spectrum. Indian J Otorhynolaryngol Head Neck Surg 1997;49:32-7.

3. Valencia MP, Castillo M. Congenital and acquired lesions of the nasal septum: A practical guide for differential diagnosis. Radio Graphics 2008;28:205-23.

4. Hedman J, Kaprio J, Poussa T et al. Prevalence of asthma, aspirin intolerance, nasal polyposis and chronic obstructive pulmonary disease in a population-based study. Int J Epidemiol 1999;28:717-22.

5. Khan N, Zafar U, Afrozi N, Ahmad SS, Hafan SA. Masses of Nasal cavity, Paranasal sinuses and Nasopharynx: A clinicopathological study. Indian J otolaryngol Head Neck Surg 2006;58:233-7.

6. Tondon PL, Gulati J, Mehta N. Histological study of polypoidal lesions in the nasal cavity. Indian J Otolaryngol 1971;13:3-11.

7. Bakari A, Afolabi OA, Adoga AA et al. Clinico-pathological profile of sinonasal masses: an experience in national ear care center Kaduna, Nigeria. BMC Research Notes 2010;3:186-96.

8. Zafar U, Khan N, Afroz N et al. Clinicopathological study of nonneoplastic lesions of nasal cavity and paranasal sinuses. Indian $\mathrm{J}$ Pathol Microbiol 2008;51:26-9.

9. Pradhananga RB, Adhikari P, Thapa NM et al. Overview of nasal masses. J Inst Med 2008;30:13-6.

10. Wischneiwski E, Klaber HG, Oppermann J. Nasal glioma as a rare cause of obstructed nasal breathing in a newborn infant. Klim padiatr 2001;213:139-41.

11. Rahbor R, Resto UA, Robson $\mathrm{CD}$ et al. Nasal glioma and encephalocele: Diagnosis and management. Laryngoscope 2003;113:2069-77.

12. Chang KC, Leu YS. Nasal glioma: A case report. Ear Nose Throat J 2001;80:410-1.

13. Soon SR, Lim CM, Singh H, Sethi DS. Sphenoid sinus mucocele: 10 cases and review of literature. J laryngol Otol 2010;124:44-7.

14. Waldman SR, Levine HL, Sebek BA. Nasal tuberculosis: A forgotten entity. Laryngoscope 1981;91:11-6.

15. Nayar RC, Al Kabi J, Ghorpade K. Primary nasal tuberculosis: A case report. Ear Nose Throat J 2004;83:188-91.

\section{CONCLUSION}

A variety of non-neoplastic and neoplastic conditions involve nasal cavity, paranasal sinuses and nasopharynx. Malignancy should be distinguished from non-malignant lesions. The presenting features, symptomatology and advanced imaging techniques help to reach a presumptive diagnosis but histopathological examination remains the mainstay of final definitive diagnosis so that a correct and timely intervention is done.

16. Syrjänen KJ. HPV infections in benign and malignant sinonasa lesions. J Clin Pathol. 2003;56:174-81.

17. Barnes L, Tse LLY, Hunt JL. Schneiderian papillomas. In: Barnes L, Eveson JV, Reichart P et al., editors. World health organization classification of tumors. Lyon: Pathology of the Head and Neck Tumors. Lyon: IARC Press;2005.pp28-32.

18. Califano J, Koch W, Sidransky D et al. Inverted sinonasal papilloma: a molecular genetic appraisal of its putative status as a precursor to squamous cell carcinoma. Am J Pathol 2000:156333-156337.

19. Lund VJ, Stammberger H, Nicolai P et al. European position paper on endoscopic management of tumours of the nose, paranasal sinuses and skull base. Rhinology. 2010;48:140-43.

20. Choi YC, Jeon EJ, Park YS. Ossifying fibroma arising in the right ethmoid sinus and nasal cavity. Int $\mathrm{J}$ Pediatr Otorhinolaryngol 2000;54:159-69.

21. Lawton MT, Heiserman JE, Coons SW et al Juvenile active ossifying fibroma: Report of four cases. J Neurosurg 1997;86:279-85.

22. Jayachandran S, Meenakshi R. Cemento-ossifying fibroma. Indian J Dent Res 2004;15:35-9.

23. A Lathi, M.M.A Syed, P. Kalakoti, D. Qutub and S.P. Kishve. Clinicopathological profile of sinonasal masses: a study from a tertiary care hospital of India. Acta Otorhinolaryngol Ital 2011;31:372-7.

24. Svane-Knudsen V, Jorgensen KE, Hansen O, et al. Cancer of the nasal cavity and paranasal sinuses: a series of 115 patients. Rhinology 1998;36:12-4.

25. Fasunla AJ, Lasisi AO. Sinonasal malignancies: A 10-year review in a tertiary health institution. J Natl Med Assoc 2007;99:1407-10.

26. Proulx GM, Caudra-Garcia I, Ferry J et al. Lymphoma of the nasal cavity and paranasal sinuses; treatment and outcome of early stage disease. Am J Clin Oncol 2003;26:252-6.

27. Akira Tamada M.D., Kazuo Makimota M.D., Masanao Okawa M.D. et al. Olfactory neuroblastoma; Presentation of a case and review of the Japanese literature. The laryngoscope 1984;94:222-5.

28. Ahmed AA, Tsokos M. Sinonasal rhabdomyosarcoma in children and young adults. Int J Surg Pathol 2007;15:160-5. 